\title{
Validity of Amontons' law for run-in short-cut aramid fiber reinforced elastomers: The effect of epoxy coated fibers
}

\author{
M. KHAFIDH ${ }^{1,2,3,}$, D. J. SCHIPPER ${ }^{1}$, M. A. MASEN ${ }^{4}$, N. VLEUGELS ${ }^{1,2}$, W. K. DIERKES ${ }^{1}$, J. W. M. NOORDERMEER ${ }^{1}$ \\ ${ }^{1}$ Faculty of Engineering Technology, University of Twente, Enschede 7500AE, The Netherlands \\ ${ }^{2}$ Dutch Polymer Institute, Eindhoven 5600AX, The Netherlands \\ ${ }^{3}$ Department of Mechanical Engineering, Universitas Islam Indonesia, Yogyakarta 55584, Indonesia \\ ${ }^{4}$ Department of Mechanical Engineering, Imperial College London, London SW72AZ, United Kingdom \\ Received: 12 September 2018 / Revised: 12 January 2019 / Accepted: 01 July 2019 \\ (C) The author(s) 2019.
}

\begin{abstract}
Friction between two contacting surfaces is studied extensively. One of the known friction theories is Amontons' law which states that the friction force is proportional to the normal force. However, Amontons' law has been found to be invalid for elastomers. In the present study, the validity of Amontons' law for short-cut aramid fiber reinforced elastomers is studied. Two types of fillers are used to reinforce the elastomers, namely highly dispersible silica and short-cut aramid fibers. Short-cut aramid fibers with two different surface treatments are used, namely non-reactive fibers with standard oily finish (SF-fibers) and fibers treated with an epoxy coating (EF-fibers). A pin-on-disc tribometer is used to investigate the frictional behavior of the composites in sliding contact with a granite counter surface. The results show that, after the run-in phase, Amontons' law is valid for those composites that are reinforced by short-cut aramid fibers (without reinforcing filler, i.e., silica) if the contact pressure is below a threshold value. However, once the contact pressure exceeds this threshold value, Amontons' law will be invalid. The threshold contact pressure of the composites containing EF-fibers is higher than of the composites containing SF-fibers. The composites that are reinforced by silica and short-cut aramid fibers do not follow Amontons' law.
\end{abstract}

Keywords: Amontons' law; elastomer; epoxy coated fiber; short-cut aramid fiber

\section{Introduction}

The sliding friction between two contacting materials has been extensively studied for many centuries. Amontons' law from the late 1,600 s states that the friction force, $F_{\mathrm{f}}$ is proportional to the normal force, $F_{\mathrm{N}}$ [1]. The ratio of friction force and normal force is called coefficient of friction, $\mu$. According to Amontons' law, the coefficient of friction does not depend on the normal force. It is also independent of the apparent (macroscopic) contact area and the relative velocity between the two contacting materials.

There are several explanations for Amontons' law, one of the explanations is the linear dependence of the frictional shear stress on the normal force. However, this explanation is not valid when surface roughness occurs on many different length scales [2]. Another explanation for Amontons' law is the proportionality of the contact area and the normal force, while the frictional shear stress is assumed to be independent of the normal force. Bowden and Tabor [3] suggested that, in many cases, only a small fraction of the surfaces are actually in contact with the contacting material because of the surface roughness. This surface roughness is assumed to be the other explanation for Amontons' law [3-5]. Amontons' law was found to be

*Corresponding author: M. KHAFIDH, E-mail: khafidh@uii.ac.id 
invalid under several conditions, such as the absence of multiple real contact points, the presence of strong adhesion, under nonlinear dependence of the real contact area with normal force, under conditions of surface detachment, and in response to a change in surface conditions [6]. Later, an analytical model to predict the dependence of coefficient of friction on normal force was studied [7].

Elastomers are used in many common applications, such as tires and conveyor belts. However, a single elastomeric material usually cannot deliver all the required properties that are needed in the aforementioned applications. Adding fibers into an elastomer is one way to improve the mechanical and the tribological properties of the composite. For an elastomeric material in contact with a counter surface, Amontons' law is known to be invalid [8]. Instead of following Amontons' law, the coefficient of friction of elastomeric materials depends on the contact pressure, sliding velocity, temperature, and lubrication regime [9]. Although Amontons' law has been investigated by many researchers, the mechanisms behind and validity of Amontons' law are still being discussed $[2,10,11]$.

In the present study, the validity of Amontons' law for short-cut aramid fiber reinforced elastomers is investigated. Four types of composite materials that are reinforced by silica and short-cut aramid fibers are used. Two types of short-cut aramid fibers with different surface treatments are used in the present study. The wear surface and the wear particles of the composites are studied to investigate the validity of Amontons' law for short-cut aramid fiber reinforced elastomers.

\section{Materials and methods}

Four types of elastomers based on a styrene butadiene rubber (SBR) and a butadiene rubber (BR) were prepared in the present study. The elastomers were reinforced by two types of reinforcing systems: highly dispersible silica and short-cut aramid fibers. Two types of poly-p-phenylene-terephtalamide (aramid) fibers were provided by Teijin Aramid B.V., Arnhem, The Netherlands: non-reactive short-cut aramid fibers with standard oily finish (SF-fibers) and short-cut aramid fibers treated with an epoxy coating (EF-fibers). The initial length of the short-cut aramid fibers was approximately $3 \mathrm{~mm}$ with a diameter of 10-12 $\mu \mathrm{m}$.

The interfacial strength between a short-cut aramid fiber and an elastomer matrix can be influenced by two elements: an adhesive coating and a coupling agent that can interact with this coating. In the present study, an epoxy coating on the fiber surface was used, which has the ability to chemically react with a coupling agent. Silane coupling agents S-3-(triethoxysilylpropyl)octanethioate (NXT) and bis-(tri-ethoxy-silyl propyl) tetrasulfide (TESPT) were used to improve the interfacial interaction between the fibers and matrix. In the present study, two systems were used: (1) shortcut aramid fiber reinforced elastomers, and (2) silica and short-cut aramid fiber reinforced elastomers. Furthermore, SF-fibers and EF-fibers were used for each system. The samples' codes that are used to describe those composites are: (1) composite $15 \mathrm{SF}$, (2) composite $15 \mathrm{EF}$, (3) composite $20 \mathrm{SF}+$ silica, and (4) composite $20 \mathrm{EF}+$ silica. The numbers 15 and 20 represent the amount of fibers in parts per hundred rubber (phr). Details of the formulation in phr are given in Table 1. Composites $15 \mathrm{SF}$ and $15 \mathrm{EF}$ are an optimized formulation based on a previous study [12], while the formulation of composites $20 \mathrm{SF}+$ silica and $20 \mathrm{EF}+$ silica is based on a silica-reinforced passenger car tire tread, branded the "Green Tire" [13].

To investigate the effect of fiber orientation of the composites containing SF-fibers and EF-fibers, four types of reinforcement directions were prepared for composites $15 \mathrm{SF}$ and $15 \mathrm{EF}$, namely randomly oriented, longitudinally oriented (meaning that the fibers are aligned in the sliding direction), transversely oriented (meaning that the fibers are aligned perpendicularly to the sliding direction) and normally oriented (meaning that the fibers are aligned perpendicularly to the composite's contact surface). The fiber direction of composites $20 \mathrm{SF}+$ silica and $20 \mathrm{EF}+$ silica are randomly oriented.

When the fibers direction are randomly oriented, the composite can be assumed to be an isotropic material. It means that the mechanical properties of the composite are the same in all directions. When the fibers are aligned in a certain direction, the mechanical properties are different in each direction so that the composite has an anisotropic behavior. Fibers in the composite are oriented during the composites' production by using a two-roll mill. Composites with 
Table 1 Material formulation of the composites.

\begin{tabular}{|c|c|c|c|c|c|}
\hline Ingredients & $\begin{array}{l}\text { Composite } 15 \mathrm{SF} \\
\text { [in phr] }\end{array}$ & $\begin{array}{l}\text { Composite } 15 \mathrm{EF} \\
\text { [in phr] }\end{array}$ & $\begin{array}{c}\text { Composite } \\
20 \mathrm{SF}+\text { silica } \\
\text { [in phr] }\end{array}$ & $\begin{array}{c}\text { Composite } \\
20 \text { EF+silica } \\
\text { [in phr }]\end{array}$ & Supplier \\
\hline $\begin{array}{c}\text { SBR, Buna VSL VP PBR } \\
4045 \mathrm{HM}\end{array}$ & 100 & 100 & - & - & $\begin{array}{c}\text { Arlanxeo, Leverkusen, } \\
\text { Germany }\end{array}$ \\
\hline SBR, Buna VSL 5025-2 HM & - & - & $97.3 *$ & $97.3 *$ & $\begin{array}{c}\text { Arlanxeo, Leverkusen, } \\
\text { Germany }\end{array}$ \\
\hline BR, KBR 01 & - & - & 30.0 & 30.0 & Kumho, Seoul, R. O. Korea \\
\hline Silica Ultrasil VN3 & - & - & 80.0 & 80.0 & $\begin{array}{c}\text { Evonik Industries AG, } \\
\text { Essen, Germany }\end{array}$ \\
\hline Zinc oxide $(\mathrm{ZnO})$ & 2.5 & 2.5 & 2.5 & 2.5 & $\begin{array}{c}\text { Sigma Aldrich, St. Louis, } \\
\text { United States }\end{array}$ \\
\hline Stearic acid (SA) & 1.5 & 1.5 & 2.5 & 2.5 & $\begin{array}{c}\text { Sigma Aldrich, St. Louis, } \\
\text { United States }\end{array}$ \\
\hline TDAE oil & - & - & 6.7 & 6.7 & $\begin{array}{l}\text { Hansen \& Rosenthal, } \\
\text { Hamburg, Germany }\end{array}$ \\
\hline Twaron aramid fiber & $15 \mathrm{SF}$ & $15 \mathrm{EF}$ & $20 \mathrm{SF}$ & $20 \mathrm{EF}$ & $\begin{array}{l}\text { Teijin Aramid B.V, } \\
\text { Arnhem, The Netherlands }\end{array}$ \\
\hline TESPT & - & - & 7.0 & 7.0 & $\begin{array}{l}\text { Evonik Industries AG, } \\
\text { Essen, Germany }\end{array}$ \\
\hline NXT & 6.0 & 6.0 & - & - & $\begin{array}{c}\text { Momentive, New York, } \\
\text { United States }\end{array}$ \\
\hline 6PPD stabilizer & - & - & 2.0 & 2.0 & Flexsys, Brussels, Belgium \\
\hline TMQ stabilizer & - & - & 2.0 & 2.0 & Flexsys, Brussels, Belgium \\
\hline Sulfur & 2.8 & 2.8 & 1.4 & 1.4 & $\begin{array}{c}\text { Sigma Aldrich, St. Louis, } \\
\text { United States }\end{array}$ \\
\hline $\begin{array}{l}\text { N-Cyclohexyl Benzothiazole } \\
\text { Sulfenamide (CBS) }\end{array}$ & 3.4 & 3.4 & 1.7 & 1.7 & Flexsys, Brussels, Belgium \\
\hline Di-Phenyl Guanidine (DPG) & 4 & 4 & 2.0 & 2.0 & Flexsys, Brussels, Belgium \\
\hline $\begin{array}{l}\text { Orientation relative to } \\
\text { the sliding contact }\end{array}$ & $\begin{array}{c}\text { Random, } \\
\text { longitudinal, } \\
\text { transverse, normal }\end{array}$ & $\begin{array}{c}\text { Random, } \\
\text { longitudinal, } \\
\text { transverse, normal }\end{array}$ & Random & Random & - \\
\hline
\end{tabular}

* Containing $37.5 \mathrm{wt} \%$ oil.

thicknesses of 2 and $5 \mathrm{~mm}$ were prepared for tensile and tribometer tests, respectively.

A pin-on-disc tribometer was used for measuring the friction force of the composites. The pin-on-disc tribometer was equipped with a rigid (compared to the elastomer) granite ball, sliding against a flat elastomeric composite disc. The granite ball with a diameter of $35 \mathrm{~mm}$ was used as a counter surface. The arithmetic average roughness of the granite ball with a cut-off length of $800 \mu \mathrm{m}$ is $1.16 \pm 0.18 \mu \mathrm{m}$. The tribometer tests were performed under dry sliding conditions at room temperature. The tests were stopped once the coefficient of friction reached a steady-state value. Three types of investigations were performed in the present study: (1) the effect of contact pressure on the frictional behavior; (2) the effect of epoxy coated fibers on the friction behavior; and (3) the breakdown of Amontons' law when applied to the short-cut aramid fibers reinforced elastomers.

A velocity of $0.2 \mathrm{~m} / \mathrm{s}$ was used for all tribometer tests. The effect of contact pressure was investigated by using two types of reinforcement system: (1) shortcut aramid fiber reinforced elastomers; and (2) silica and short-cut aramid fiber reinforced elastomers. The composites used for this investigation were $15 \mathrm{SF}$ and $20 \mathrm{SF}+$ silica. Two contact pressures were used for every composite, namely 0.15 and $0.20 \mathrm{MPa}$ for composites $15 \mathrm{SF}$, and 0.68 and $0.8 \mathrm{MPa}$ for composite $20 \mathrm{SF}+$ silica. The effect of epoxy coated fibers was evaluated by comparing those composites that are reinforced by 
EF-fibers and SF-fibers for both formulations. A contact pressure of $0.2 \mathrm{MPa}$ was used to compare the friction behavior between the composites $15 \mathrm{SF}$ and $15 \mathrm{EF}$, while the comparison between composites $20 \mathrm{SF}+$ silica and $20 \mathrm{EF}+$ silica was conducted using a contact pressure of $0.68 \mathrm{MPa}$. Based on the results of those investigations, the breakdown of Amontons' law was investigated by further varying the contact pressure. The normal load was adapted, depending on the reinforcement direction of the fibers based on the anisotropic-viscoealastic contact model [14].

A Keyence VHX-5000 microscope and a Jeol JSM 6400 scanning electron microscope (SEM) were used to scan images of the wear surfaces and the wear particles. Tensile measurements were performed with an Instron tensile tester 3343 series, according to ISO 37 at a crosshead speed of $500 \mathrm{~mm} / \mathrm{min}$.

\section{Results}

\subsection{Mechanical properties of the materials}

One of the important factors that influences the mechanical properties of short-cut aramid fiber reinforced elastomers is the interfacial interaction between the fibers and the elastomer matrix. An epoxy coating on the fiber surface, in combination with a silane coupling agent, may improve that interaction. The stress-strain relations of all composites with randomly oriented fibers are shown in Fig. 1. The lack of silica as reinforcing filler in the composite leads to a large reduction in the failure point of the tensile stressstrain test. A yield point is observed for the composites containing silica and short-cut aramid fibers (composites $20 \mathrm{SF}+$ silica and $20 \mathrm{EF}+$ silica) at a strain of approximately $30 \%$. At the strain below the yield, the composites containing silica and short-cut aramid fibers show a steeper curve compared to those composites containing solely short-cut aramid fibers. Moreover, a steeper curve is found for the composites containing EF-fibers compared to those containing SF-fibers. It confirms that an epoxy coating on the fiber surfaces in combination with a coupling agent is effective in improving the mechanical properties of the composites.

Figure 2 shows the stress-strain relations of composites $15 \mathrm{SF}$ and $15 \mathrm{EF}$ for several reinforcement

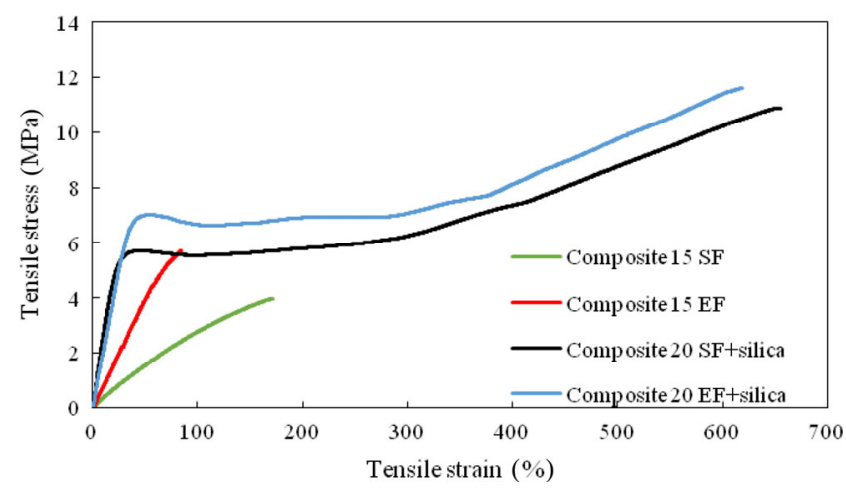

Fig. 1 Stress-strain relations of all composites with randomly oriented fibers.

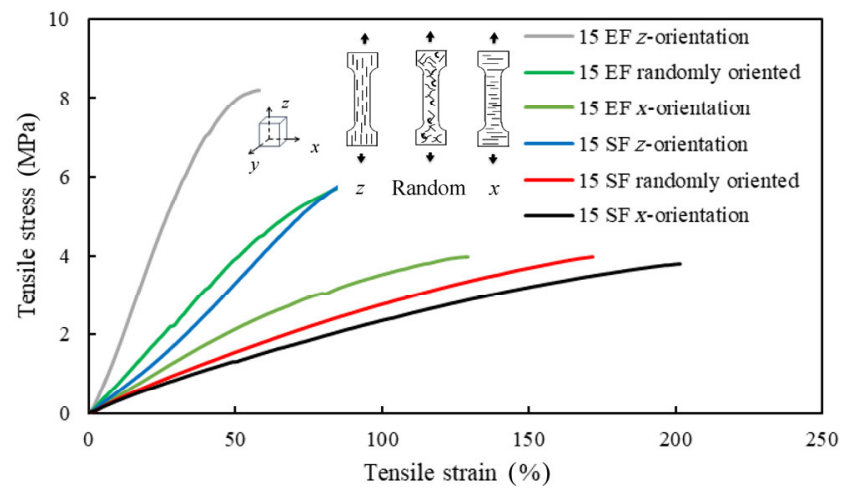

Fig. 2 Stress-strain relations of composites 15 SF and 15 EF for several reinforcement directions.

directions. As expected, the composite reinforced in the $z$-direction (the fibers are aligned longitudinally to the applied force) has the steepest curve whereas the composite reinforced in the $x$-direction (the fibers are aligned perpendicularly to the applied force) shows the shallowest curve, while the composite with randomly oriented fibers shows an intermediate slope. The composites containing EF-fibers have a steeper curve compared to that for the composites containing SF-fibers for all reinforcement directions. The elastic moduli were defined at a strain of $2 \%$, assuming the material behaves linearly below that strain value [15].

\subsection{Effect of contact pressure}

Figure 3(a) shows the coefficients of friction as a function of sliding distance for composite $15 \mathrm{SF}$ at two different contact pressures, namely 0.15 and $0.20 \mathrm{MPa}$. It can be seen that the steady-state coefficients of friction of those composites decrease to approximately 4 times lower than that at the beginning of the tests. The reason of this phenomenon is that a part of the fibers 

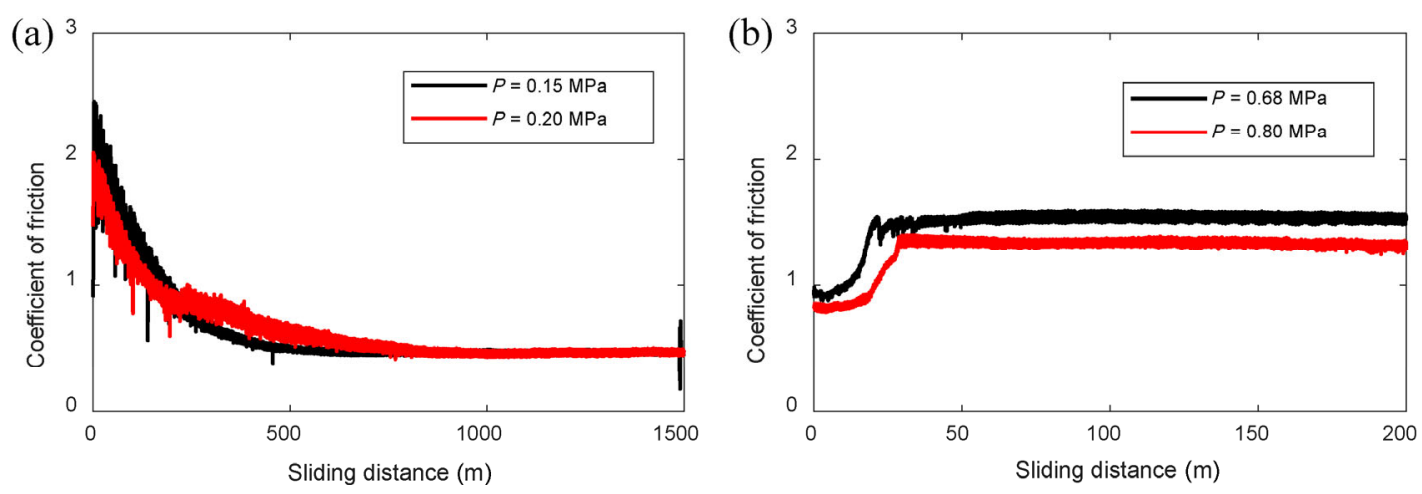

Fig. 3 Coefficients of friction as a function of sliding distance for different contact pressures: (a) composite 15 SF and (b) composite $20 \mathrm{SF}+$ silica. All composites have randomly oriented fibers.

are sticking out of the composite and they are present on the wear surface $[16,17]$. It leads to a reduction of the real contact area between the counter surface and the composite. Although the coefficients of friction are different at the beginning of the tests, the steady-state coefficients of friction values are the same for both contact pressures.

Figure 3(b) shows the coefficients of friction as a function of sliding distance for composite $20 \mathrm{SF}+$ silica at two different contact pressures, namely 0.68 and $0.80 \mathrm{MPa}$. A higher contact pressure results in a lower coefficient of friction. The decreasing coefficient of friction as seen in Fig. 3(a) does not feature in these tests. Moreover, the coefficient of friction reaches a steady-state signal at a short sliding distance of $\sim 30 \mathrm{~m}$.

The wear surfaces of the composites after the tribometer tests are shown in Fig. 4. The wear surfaces of composite $15 \mathrm{SF}$ at both contact pressures have the same characteristic, in which many fibers exist on the wear surface and align in the direction of sliding, see Fig. 4(a). In a previous article [16], it was shown that the coefficient of friction of elastomers that are reinforced with a high amount of short-cut aramid fibers is controlled by the fibers on the wear surface rather than by the bulk properties. Since the counter surface is mainly in contact with the fibers, the steadystate coefficients of friction of composite $15 \mathrm{SF}$ at both contact pressures have a uniform value.

The wear surface of composite $20 \mathrm{SF}+$ silica at a contact pressure of $0.8 \mathrm{MPa}$ has the same characteristic as that at a contact pressure of $0.68 \mathrm{MPa}$, where only a few fibers are observed on the wear surface. Figure 4(b) shows the wear surface of composite $20 \mathrm{SF}+$ silica at a contact pressure of $0.8 \mathrm{MPa}$. Since only a few fibers are present on the wear surface, the counter surface is mainly in contact with the bulk material. Hence, the coefficient of friction is mainly influenced by the bulk properties of the composite.

\subsection{Effect of epoxy coating}

Interfacial interaction between the short-cut aramid fiber and the elastomer matrix plays an important
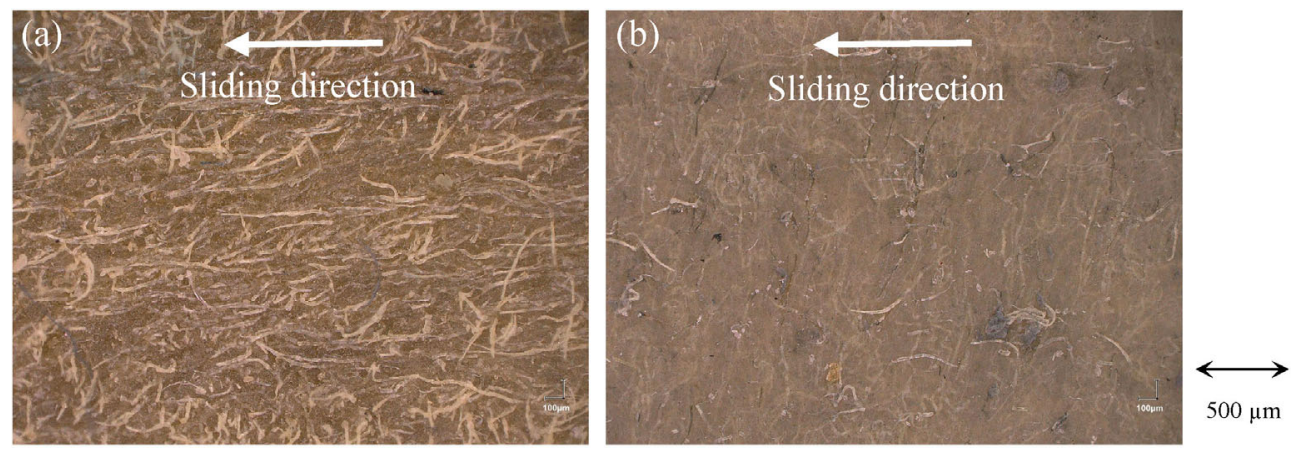

Fig. 4 Wear surfaces after tribometer tests: (a) composite $15 \mathrm{SF}$ at a contact pressure of $0.2 \mathrm{MPa}$ and (b) composite $20 \mathrm{SF}+$ silica at a contact pressure of $0.8 \mathrm{MPa}$. 
role in reinforcing the composite and controls the mechanical properties. Therefore, an investigation into the effects of surface treatment on the fiber surface is important in improving the mechanical properties and also the tribological behavior of the composites. Figures 1 and 2 show that by adding EF-fibers to the composite leads to an improvement of the mechanical properties compared to those of the composite containing SF-fibers.

The coefficients of friction as a function of sliding distance for composites $15 \mathrm{SF}$ and $15 \mathrm{EF}$ in random orientation at a contact pressure of $0.2 \mathrm{MPa}$ are shown in Fig. 5(a). It can be seen that after a sliding distance of approximately $800 \mathrm{~m}$, the coefficients of friction reach the same steady-state values, namely $\sim 0.5$. Figure $5(b)$ shows the coefficients of friction as a function of sliding distance for composites $20 \mathrm{SF}+$ silica and $20 \mathrm{EF}+$ silica in random orientation at a contact pressure of $0.68 \mathrm{MPa}$. The coefficients of friction reach a steady-state phase after a short sliding distance, namely $\sim 30 \mathrm{~m}$. The composite containing EF-fibers (composite $20 \mathrm{EF}+$ silica) has a slightly lower steady-state coefficient of friction compared to that containing SF-fibers (composite $20 \mathrm{SF}+$ silica). This may be attributed to the fact that the mechanical properties of composite $20 \mathrm{EF}+$ silica are higher than that of composite $20 \mathrm{SF}+$ silica. Therefore, the contact area of composite $20 \mathrm{EF}+$ silica is smaller than that of composite $20 \mathrm{SF}+$ silica. These results show that the coefficient of friction of the short-cut aramid fiber reinforced elastomer (without silica reinforcement) is independent of the mechanical properties of the composite, while the mechanical properties of silica and short-cut aramid fiber reinforced elastomer influence the coefficient of friction.

The wear surfaces of the composites after tribometer tests are shown in Fig. 6. It can be seen that the wear surfaces of composites $15 \mathrm{SF}$ and $15 \mathrm{EF}$ are dominated by fibers. Thus, the counter surface is mainly in contact with the fibers, and as a result, the steady-state coefficients of friction of these composites are similar. However, the length of the fibers that are present on the wear surface of composite $15 \mathrm{SF}$ is longer than that of composite $15 \mathrm{EF}$, see Figs. 6(a) and 6(b). This is caused by the interaction between the EF-fibers and the matrix which is stronger than for the SF-fibers and the matrix. Therefore, the EF-fibers are more difficult to be pulled-out and the wear resistance of composites containing EF-fibers is better than that of composites containing SF-fibers, as reported in a previous study [12].

Figures 6(c) and 6(d) show the wear surfaces of composites $20 \mathrm{SF}+$ silica and $20 \mathrm{EF}+$ silica, respectively. Although the fiber amount of composites $20 \mathrm{SF}+$ silica and $20 \mathrm{EF}+$ silica are higher than for composites $15 \mathrm{SF}$ and $15 \mathrm{EF}$, the fibers which exist on the wear surfaceare far less. This suggests that the composites containing silica and short-cut aramid fibers can avoid the elastomer matrix pull-out and fiber detachment effectively. Since the contact mainly occurs between the counter surface and the bulk material, the coefficient of friction depends on the bulk properties of the composite. Hence, the coefficient of friction of the "stiffer" material (composite $20 \mathrm{EF}+$ silica) is lower than of the weaker material (composite $20 \mathrm{SF}+$ silica).
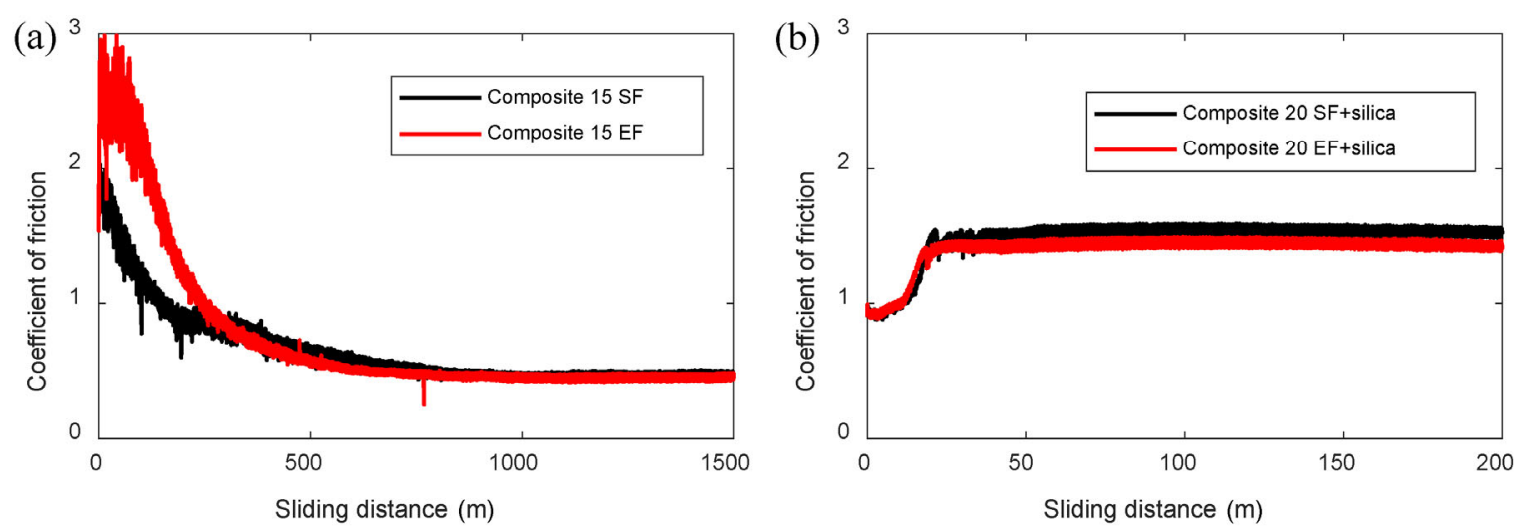

Fig. 5 Coefficients of friction as a function of sliding distance for: (a) composite 15 SF vs. composite 15 EF at a contact pressure of $0.2 \mathrm{MPa}$, (b) composite $20 \mathrm{SF}+$ silica vs. composite $20 \mathrm{EF}+$ silica at a contact pressure of $0.68 \mathrm{MPa}$. All composites have randomly oriented fibers. 

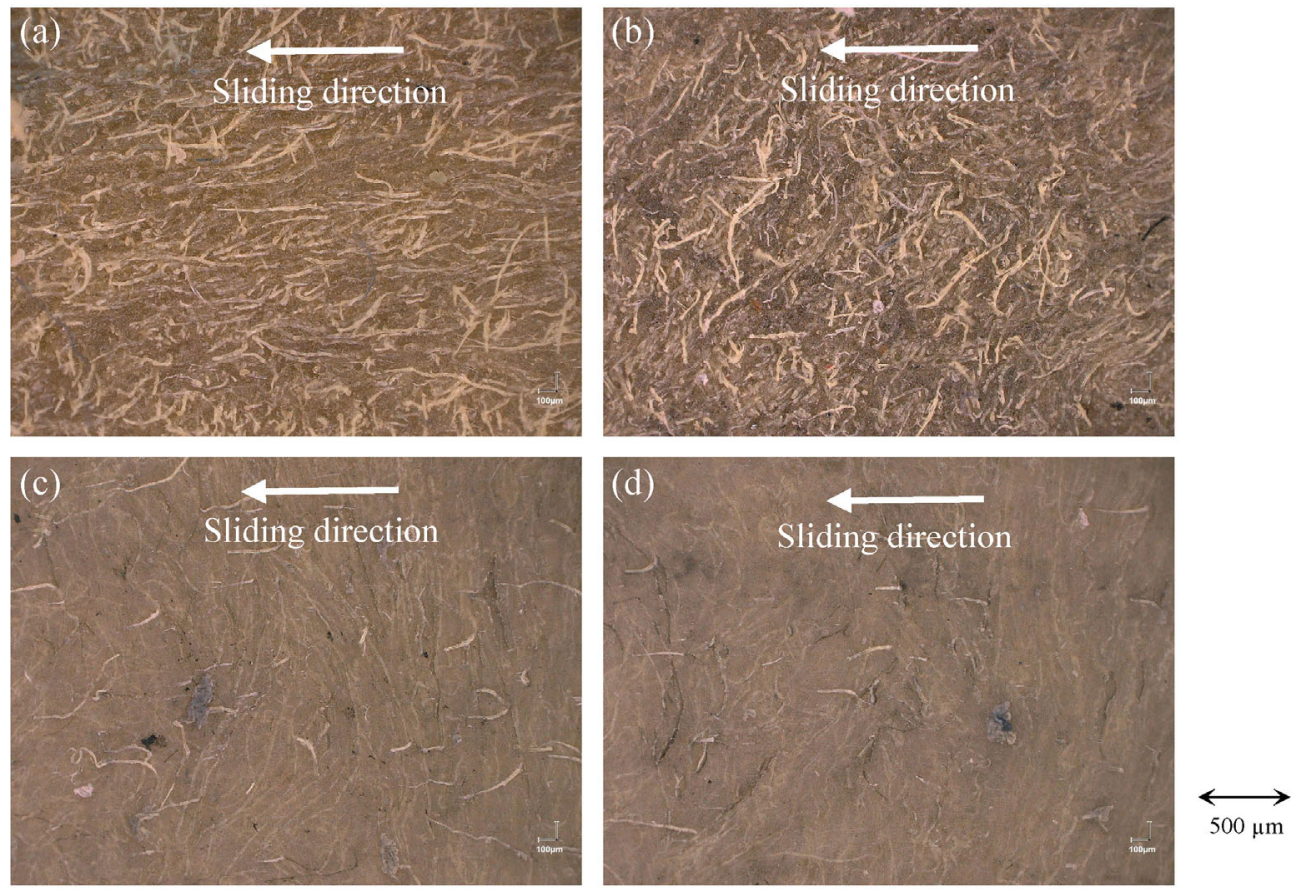

Fig. 6 Wear surfaces after tribometer tests: (a) composite $15 \mathrm{SF}$ at a contact pressure of $0.2 \mathrm{MPa}$, (b) composite $15 \mathrm{EF}$ at a contact pressure of $0.2 \mathrm{MPa}$, (c) composite $20 \mathrm{SF}+$ silica at a contact pressure of $0.68 \mathrm{MPa}$, and (d) composite $20 \mathrm{EF}+$ silica at a contact pressure of $0.68 \mathrm{MPa}$.

\subsection{Breakdown of Amontons' law}

Figure 7 shows the coefficients of friction as a function of sliding distance for composites $15 \mathrm{SF}$ and $15 \mathrm{EF}$ for several reinforcement directions at a contact pressure of $0.2 \mathrm{MPa}$. It can be seen that the coefficients of friction of all the composites show different values at the beginning of the test. After a certain sliding distance, the coefficients of friction show the same value, namely $\sim 0.5$. This suggests that the coefficient of friction is independent of the reinforcement direction.

The steady-state coefficient of friction of short-cut aramid fiber reinforced elastomer (without silica

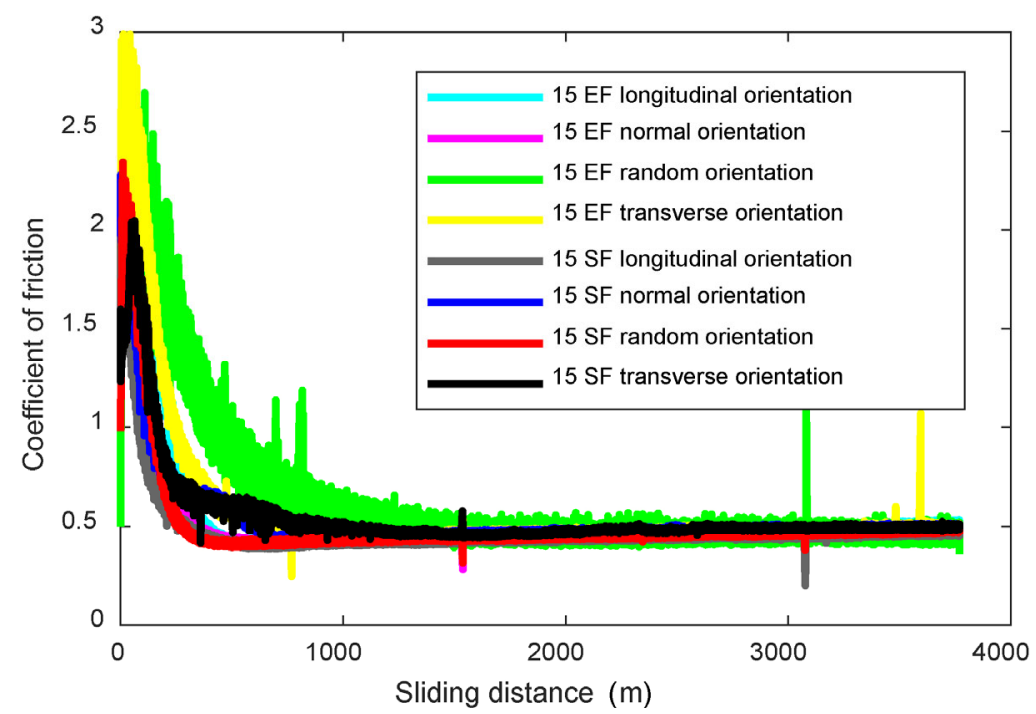

Fig. 7 Coefficients of friction as a function of sliding distance for composites $15 \mathrm{SF}$ and $15 \mathrm{EF}$ for several reinforcement directions. 
reinforcement) has been observed to be independent of the contact pressure (Fig. 3(a)), mechanical properties (Fig. 5(a)), and the fiber reinforcement direction (Fig. 7). In other words, the short-cut aramid fiber reinforced elastomers follow Amontons' law after the run-in phase. However, those composites that are reinforced by silica and short-cut aramid fibers (composites $20 \mathrm{SF}+$ silica and $20 \mathrm{EF}+$ silica) do not follow Amontons' law. The coefficients of friction of those composites depend on the contact pressure (Fig. 3(b)) and the mechanical properties of the composite (Fig. 5(b)).

The investigation of the breakdown of Amontons' law for the short-cut aramid fiber reinforced elastomers was conducted by further varying the contact pressure. Figure 8 shows the coefficients of friction as a function of sliding distance for the EF-fiber reinforced elastomers (composite $15 \mathrm{EF}$ ) at various contact pressures. It can be seen that the steady-state coefficients of friction

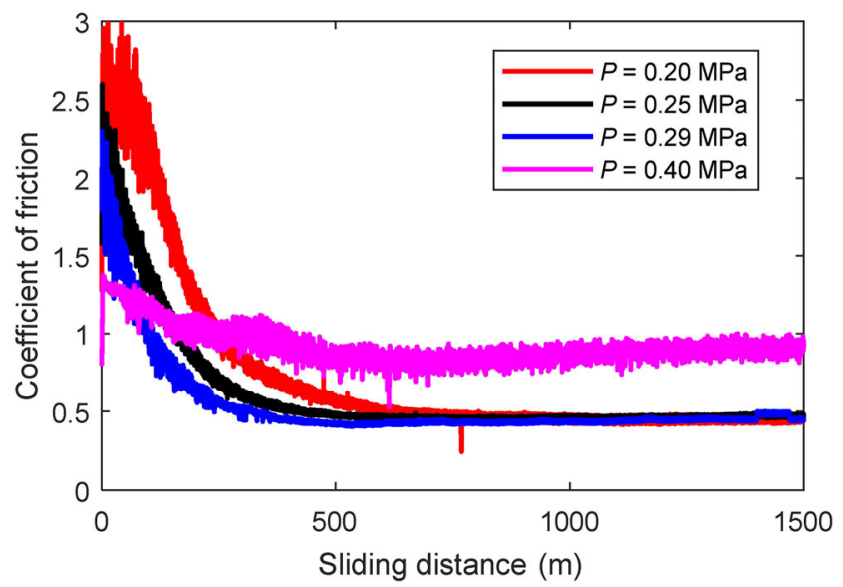

Fig. 8 Coefficients of friction as a function of sliding distance for the composite $15 \mathrm{EF}$ at different contact pressures.

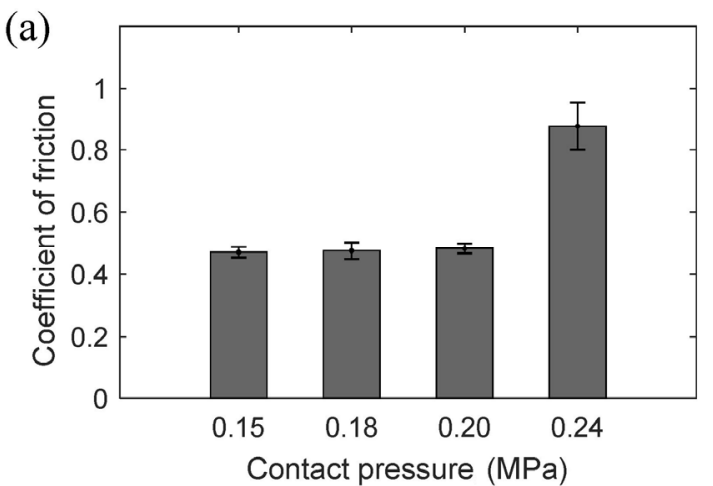

are uniform whether contact pressures of $0.2,0.25$ or $0.29 \mathrm{MPa}$ are applied, while the steady-state coefficient of friction changes when a contact pressure of $0.4 \mathrm{MPa}$ is applied. Therefore, short-cut aramid fiber reinforced elastomers follow Amontons' law as long as the contact pressure does not exceed a threshold value. Once the contact pressure exceeds that threshold, Amontons' law is not valid anymore.

Similarly, pin-on-disc tribometer tests at various contact pressures were also conducted for the SF-fiber reinforced elastomers (composite $15 \mathrm{SF}$ ). Figure 9 shows the coefficients of friction at the steady-state phase for composites $15 \mathrm{SF}$ and $15 \mathrm{EF}$. It can be seen that both composites have a threshold contact pressure, at which Amontons' law is not valid when the contact pressure exceeds that threshold. For composite $15 \mathrm{SF}$, Amontons' law is not valid when a contact pressure of $0.24 \mathrm{MPa}$ is applied, whereas Amontons' law is not valid when a contact pressure of $0.40 \mathrm{MPa}$ is applied to composite $15 \mathrm{EF}$.

The wear surfaces of the composites at the end of the tribometer tests in which Amontons' law is valid and invalid are shown in Fig. 10. Figures 10(a) and 10(b) show the wear surfaces of composite $15 \mathrm{SF}$ at a contact pressure of 0.20 and $0.24 \mathrm{MPa}$, respectively. When many fibers are present on the wear surface and align in the direction of sliding, the coefficient of friction follows Amontons' law, see Fig. 10(a). Once the contact pressure exceeds the threshold, many fibers are pulled-out from the composite, and severe wear is found. Hence, fewer fibers are present on the wear surface, see Fig. 10(b).

It was observed that the threshold contact pressure of composite $15 \mathrm{EF}$ is higher than that for composite

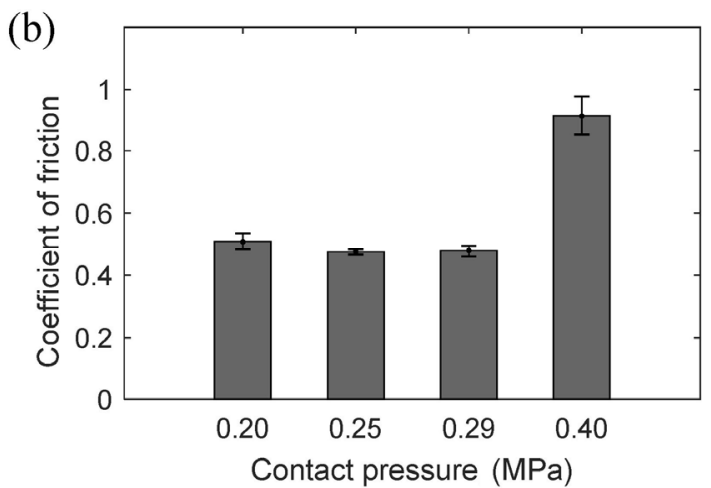

Fig. 9 Steady-state coefficients of friction at various contact pressures: (a) composite 15 SF and (b) composite 15 EF. 

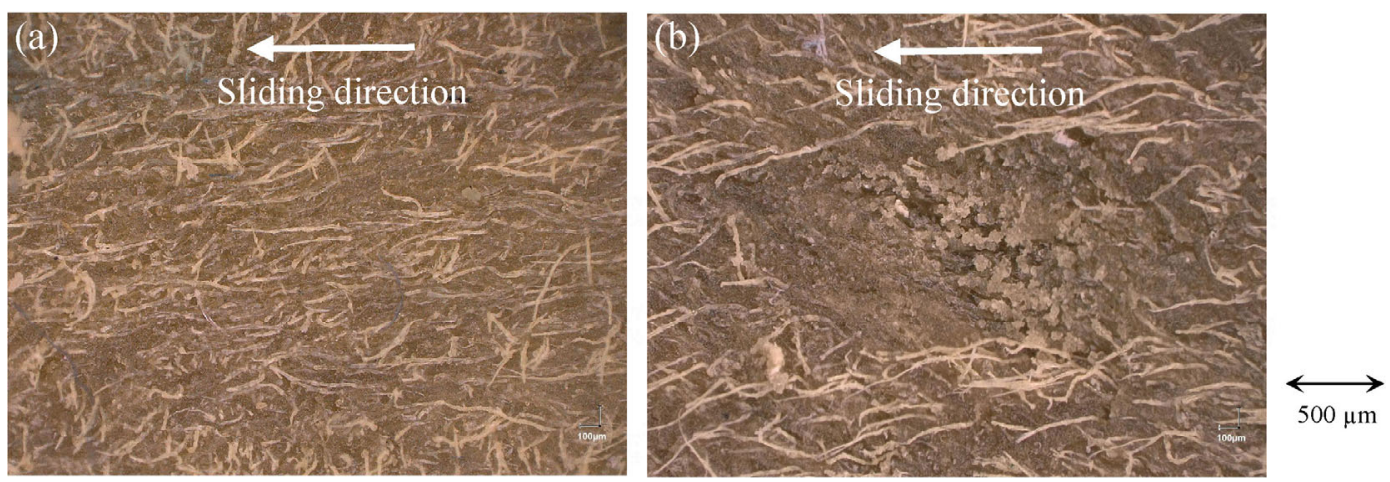

Fig. 10 Wear surfaces after tribometer tests: (a) composite $15 \mathrm{SF}$ when the Amontons' law is valid (contact pressure of $0.20 \mathrm{MPa}$ ) and (b) composite $15 \mathrm{SF}$ when the Amontons' law is not valid (contact pressure of $0.24 \mathrm{MPa}$ ).

$15 \mathrm{SF}$. This can be explained by the different interfacial interaction between the fibers and elastomer matrix of those composites. The EF-fibers result in a better interfacial interaction with an elastomer matrix compared to the SF-fibers, as shown in the mechanical properties of these composites, see Figs. 1 and 2. Therefore, a higher contact pressure is required to pull-out many fibers as shown for composite $15 \mathrm{SF}$ in Fig. 10(b).

Studying the wear particles of the composites in case Amontons' law is valid or invalid shows no clear difference. The fibers are sticking together with the elastomer matrix, only asmall part of the fibers stay apart from the elastomer matrix, see Figs. 11(a) and 11(b). While the wear particles of the composites that are reinforced by silica and short-cut aramid fibers are dominated by fibers, only a small amount of the elastomer matrix is observed in the wear particles, see Fig. 11(c). By adding silica to the composite, the propagation of cracks will be stopped when it reaches
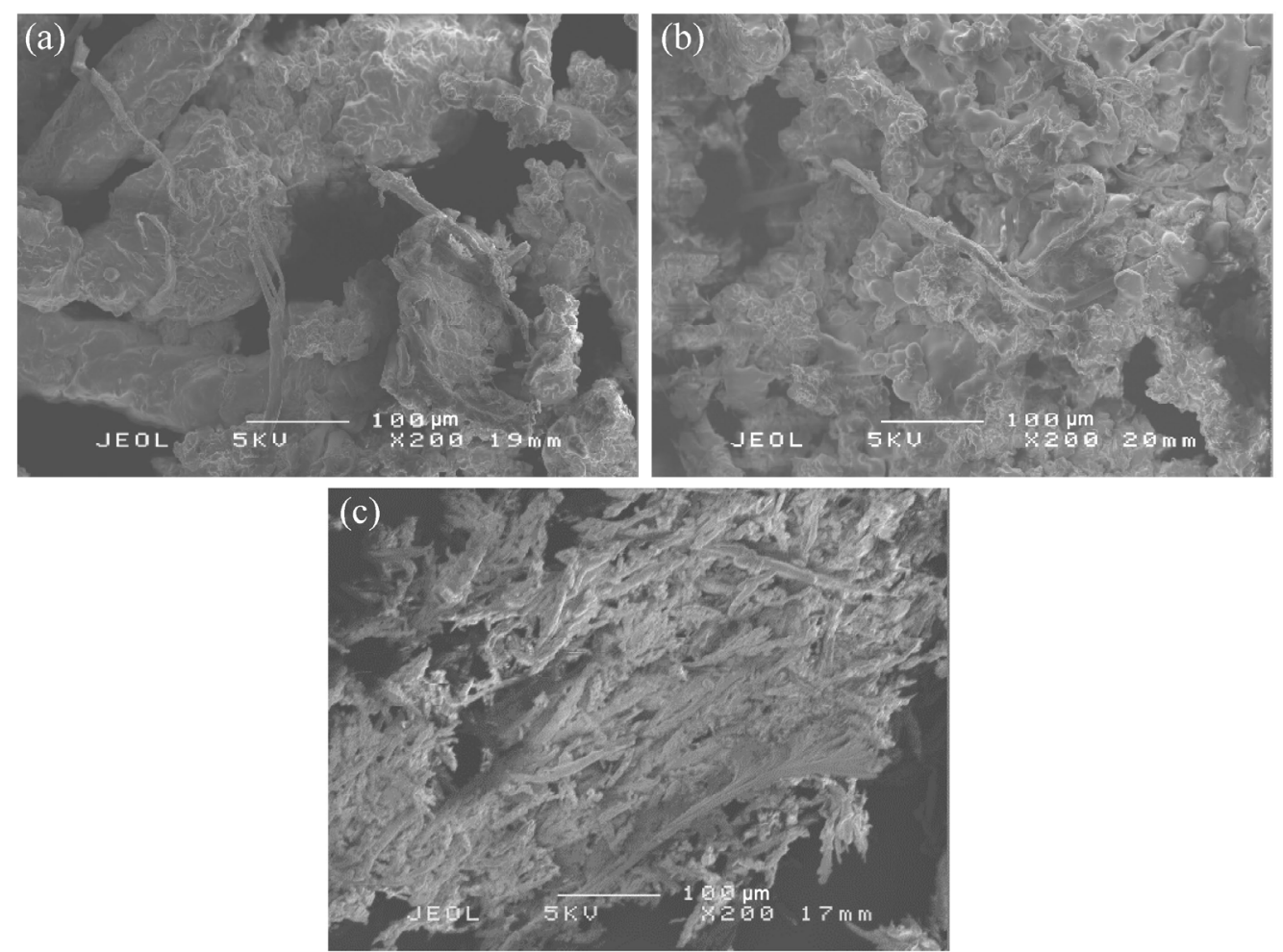

Fig. 11 SEM images of wear particles: (a) composite $15 \mathrm{SF}$ when the Amontons' law is valid (contact pressure of $0.20 \mathrm{MPa}$ ), (b) composite $15 \mathrm{SF}$ when the Amontons' law is not valid (contact pressure of $0.24 \mathrm{MPa}$ ), and (c) composite $20 \mathrm{SF}+\mathrm{silica}$ at a contact pressure of $0.80 \mathrm{MPa}$. 
the silica particle [18]. Since the propagation of cracks is stopped at short propagation and it bends to the elastomer surface, only a small amount of the elastomer matrix is pulled-out from the composite. Furthermore, the fibers that align parallel to the elastomer surface are easy to be pulled-out, whereas the fibers which are deeply embedded in the matrix give greater resistance to detach, only a small part of the fibers is sticking out of the composite are present on the wear surface, see Fig. 4(b). Many fibers in the wear particles of the silica and short-cut aramid fiber reinforced elastomers are generated by the fibers pull-out from the composite.

\section{Discussion}

It was known that Amontons' law is not valid for rubber-like materials. However, after the run-in phase, Amontons' law was found to be valid for short-cut aramid fiber reinforced elastomers (without silica reinforcement) below a threshold contact pressure. The proportionality between the real contact area and the normal force may be the source of this phenomenon. For plastic materials, plastic flow in the asperity contact region explains the proportionality between the real contact area and the normal force [2]. However, for elastomers, the local pressure in the asperity contact regions reaches the rupture stress. Therefore, plastic flow is not the explanation of Amontons' law in the case of short-cut aramid fiber reinforced elastomers without further silica reinforcement.

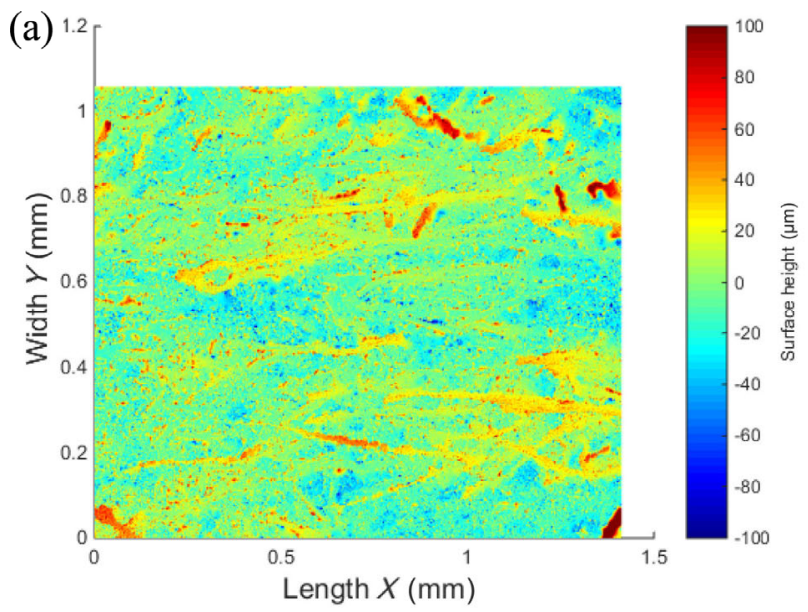

The proportionality between the real contact area and the normal force of short-cut aramid fiber reinforced elastomers may be caused by the small ratio of the real and the apparent contact area. Persson suggested that the normal force is proportional to the real contact area when the ratio between the real and the apparent contact area is less than 10\% [19]. Figure 12 shows the height of wear surfaces of short-cut aramid fiber reinforced elastomer when Amontons' law is both valid and invalid. It shows that a rough wear surface is found when Amontons' law is valid. However, a relatively smooth surface is found when the Amontons' law is not valid. Amontons' law is generally valid for a multi-contact situation, even in the case of elastomers $[7,20]$. However, Amontons' law is not valid for a contact situation of mono-contact because the friction force is usually not proportional to the normal force, for example, in the Johnson-Kendall-Roberts (JKR) sphere-plane contact [21].

Figure 13 shows the granite counter surfaces before and after the tests. It can be seen that wear debris fill the cavities of the counter surface, only elastomer debris are attached on the counter surface, whereas no fibers are observed on the counter surface, see Fig. 13(b). Since the counter surface is mainly in contact with the fibers after the run-in phase, the steady-state coefficient of friction is influenced by the contact between the elastomer debris and the fibers at the asperities. To quantify the effect of the presence of elastomer debris on the granite counter surface to the value of the steady-state coefficient of friction,

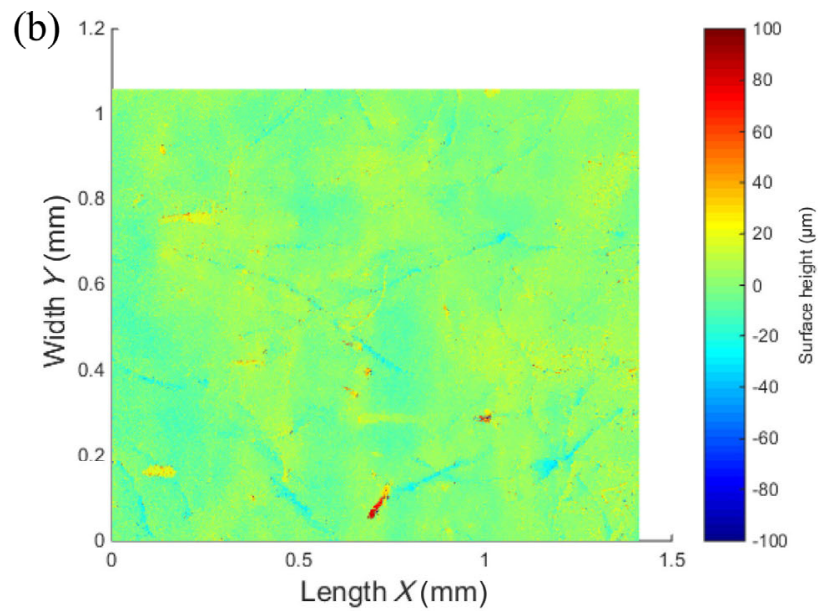

Fig. 12 Height of wear surfaces: (a) when Amontons' law is valid (composite 15 SF at a contact pressure of $0.20 \mathrm{MPa}$ ), (b) when Amontons' law is not valid (composite $20 \mathrm{SF}+$ silica at a contact pressure of $0.80 \mathrm{MPa}$ ). 
additional experiments were conducted with a "worn" elastomer surface in contact with a clean granite counter surface. Several contact pressures at which Amontons' law is valid were used. The results show that the measured steady-state coefficients of friction are similar for all tests, namely $\sim 0.3$, for instance see Fig. 14(a). These results suggest that Amontons' law is also valid for a clean granite counter surface. The presence of elastomer debris on the counter surface acts as an additional friction factor. When a clean counter surface is used, the steady-state coefficient of friction decreases from 0.5 to 0.3 in comparison with the "polluted" counter surface. The elastomer debris that is attached on the counter surface reduces the roughness and therefore increases the coefficient of friction. For the tests of "worn" composites in contact with a clean counter surface, no debris material is found on the counter surface after the test because the counter surface is mainly in contact with the fibers, see Fig. 14(b).

It has been observed that Amontons' law is not valid for composites that are reinforced by silica and short-cut aramid fibers. For smooth surfaces and soft materials, the relation between the contact area and the normal force follows the Hertz theory, $A \sim F^{2 / 3}$ or $\mu \sim F^{-1 / 3}$. For silica and short-cut aramid fiber reinforced elastomers, the relation is located somewhere in between the Hertz theory $\left(\mu \sim F^{-1 / 3}\right)$ and Amontons' theory $(\mu \sim F)$. For example, composite $20 \mathrm{SF}+$ silica has a steady-state coefficient of friction of $1.53 \pm 0.04$ at a contact pressure of $0.68 \mathrm{MPa}(F=5 \mathrm{~N})$. When a contact pressure of $0.8 \mathrm{MPa}(F=8.2 \mathrm{~N})$ is used, the steady-state coefficient of friction decreases to become $1.35 \pm 0.05$, see Fig. 3 (b). These results show that the coefficient of friction decreases with increasing normal force, but the reduction of the coefficient of friction is lower than predicted by the Hertz theory.

Although the decreasing coefficient of friction phenomenon does not occur in composites that are reinforced by silica and short-cut aramid fibers, this phenomenon may occur when a high energy input (high contact pressure and high velocity) is applied.

\section{(a)}

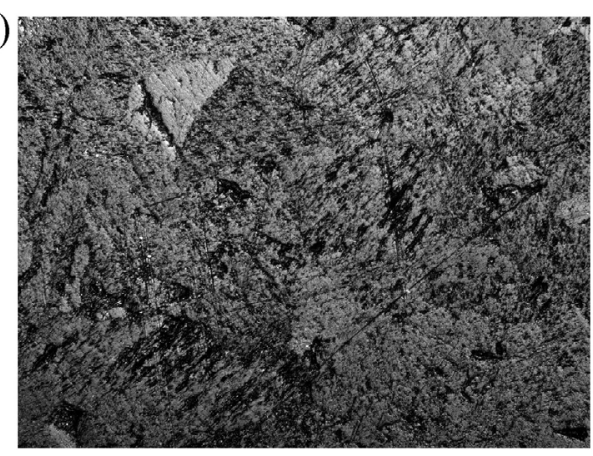

(b)

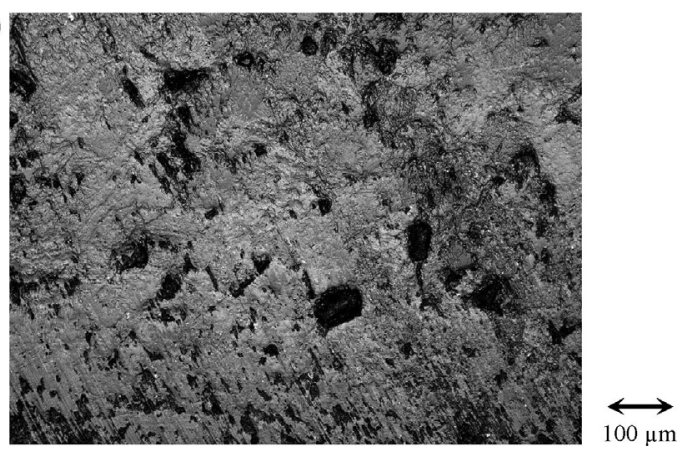

Fig. 13 Images of the granite counter surface on an area of $660 \mu \mathrm{m} \times 877 \mu \mathrm{m}$ : (a) clean counter surface, (b) "polluted" counter surface is found after the test of composite $15 \mathrm{EF}$ at a contact pressure of $0.25 \mathrm{MPa}$.

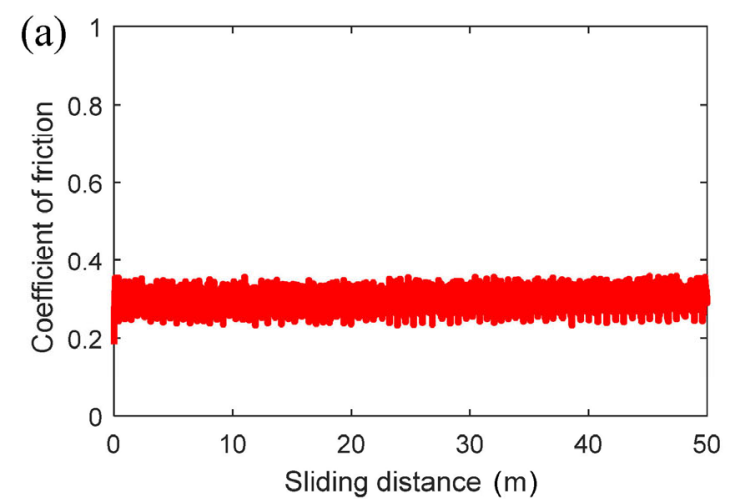

(b)

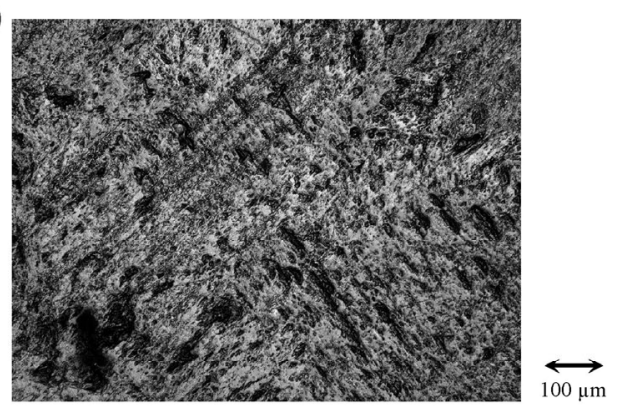

Fig. 14 (a) Coefficient of friction of "worn" composite $15 \mathrm{EF}$ at a contact pressure of $0.25 \mathrm{MPa}$ in contact with a clean granite counter surface, (b) counter surface after the test with the "worn" composite $15 \mathrm{EF}$ on an area of $660 \mu \mathrm{m} \times 877 \mu \mathrm{m}$. 
In that situation, the propagation of cracks can break the silica-matrix bond. As a result, a high amount of elastomer matrix will pull-out from the composite, and many fibers are present on the wear surface. The decreasing coefficient of friction is caused by many fibers existing on the wear surface. Therefore, the composites that are reinforced by silica and short-cut aramid fiber may follow Amontons' law when a high enough energy input is applied.

\section{Conclusions}

The validity of Amontons' law for short-cut aramid fiber reinforced elastomer has been investigated. Two types of fibers were evaluated: SF-fibers and EF-fibers. After the run-in phase, Amontons' law was found to be valid for the short-cut aramid fiber reinforced elastomer (without silica reinforcement) at contact pressures below a certain threshold value. Once the contact pressures exceed the threshold, Amontons' law is not valid anymore. Amontons' law was found to be invalid for composites that are reinforced by silica and short-cut aramid fibers. The EF-fiber reinforced elastomer has a higher threshold contact pressure than that for SF-fiber reinforced elastomers. This is caused by the surface interaction between EF-fibers and elastomer matrix which is better than that between SF-fibers and elastomer matrix. Therefore, a higher contact pressure is needed to pull-out many fibers from the composite containing EF-fibers.

\section{Acknowledgements}

This work is part of the Research Program of the Dutch Polymer Institute DPI, The Netherlands (Project No. \#782).

Open Access This article is licensed under a Creative Commons Attribution 4.0 International Li-cense, which permits use, sharing, adaptation, distribution and reproduction in any medium or for-mat, as long as you give appropriate credit to the original author(s) and the source, provide a link to the Creative Commons licence, and indicate if changes were made.

The images or other third party material in this article are included in the article's Creative Commons licence, unless indicated otherwise in a credit line to the material. If material is not in-cluded in the article's Creative Commons licence and your intended use is not permitted by statutory regulation or exceeds the permitted use, you will need to obtain permission directly from the copyright holder.

To view a copy of this licence, visit http:// creativecommons.org/licenses/by/4.0/.

\section{References}

[1] Popova E, Popov V L. The research works of Coulomb and Amontons and generalized laws of friction. Friction 3(2): 183-190 (2015)

[2] Persson B N J, Sivebæk I M, Samoilov V N, Zhao K, Volokitin A I, Zhang Z Y. On the origin of Amonton's friction law. J Phys: Condens Matter 20(39): 395006 (2008)

[3] Bowden F P, Tabor D. Friction and Lubrication of Solids. Oxford (UK): Clarendon Press, 1950.

[4] Burton Z, Bhushan B. Hydrophobicity, adhesion, and friction properties of nanopatterned polymers and scale dependence for micro- and nanoelectromechanical systems. Nano Lett 5(8): 1607-1613 (2005)

[5] Baumberger T, Caroli C. Solid friction from stick-slip down to pinning and aging. Adv Phys 55(3-4): 279-348 (2006)

[6] Otsuki M, Matsukawa H. Systematic breakdown of Amontons' law of friction for an elastic object locally obeying Amontons' law. Sci Rep 3: 1586 (2013)

[7] Maegawa S, Itoigawa F, Nakamura T. Effect of normal load on friction coefficient for sliding contact between rough rubber surface and rigid smooth plane. Tribol Int 92: 335-343 (2015)

[8] Schallamach A. Friction and abrasion of rubber. Wear 1(5): 384-417 (1958)

[9] Jiménez M A, Bielsa J M, Rodríguez R, Dobón S. The influence of contact pressure on the dynamic friction coefficient in cylindrical rubber-metal contact geometries. In IUTAM Symposium on Computational Methods in Contact Mechanics. Wriggers P, Nackenhorst U, Eds. Dordrecht: Springer, 2007

[10] Gao J P, Luedtke W D, Gourdon D, Ruths M, Israelachvili J N, Landman U. Frictional forces and Amontons' law: From the molecular to the macroscopic scale. J Phys Chem B 108(11): 3410-3425 (2004)

[11] Müser M H, Wenning L, Robbins M O. Simple microscopic theory of Amontons's laws for static friction. Phys Rev Lett 86(7): 1295-1298 (2001) 
[12] Vleugels N. Short fibre-reinforced elastomeric composites, fundamental routes towards improvement of the interfacial interaction of short-cut aramid fibres in a SBR compound, to improve friction and wear properties. Master's thesis. Enschede (The Netherlands): University of Twente, 2017.

[13] Rauline R. Composition de caoutchouc et enveloppes de pneumatiques à base de ladite composition. Patent EP 0501227, 1992.

[14] Rodriguez N V, Masen M A, Schipper D J. A contact model for orthotropic-viscoelastic materials. Int $J$ Mech Sci 74: 91-98 (2013)

[15] Hintze C, Boldt R, Wiessner S, Heinrich G. Influence of processing on morphology in short aramid fiber reinforced elastomer compounds. J Appl Polym Sci 130(3): 1682-1690 (2013)

[16] Khafidh M, Schipper D J, Masen M A, Vleugels N, Noordermeer J W M. Tribological behavior of short-cut

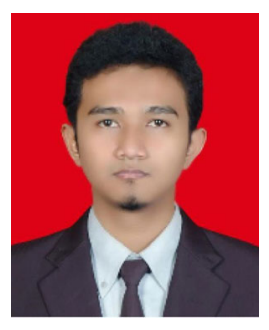

M. KHAFIDH. He received his bachelor and master degrees in mechanical engineering from University of Diponegoro in 2012 and 2013, respectively. In 2019, he aramid fiber reinforced SBR elastomers: The effect of fiber orientation. J Mech Eng Sci 12(2): 3700-3711 (2018)

[17] Khafidh M, Schipper D J, Masen M A, Vleugels N, Dierkes W K, Noordermeer J W M. Friction and wear mechanism of short-cut aramid fiber and silica reinforced elastomers. Wear 428-429: 481-487 (2019)

[18] Persson B N J. Theory of powdery rubber wear. J Phys: Condens Matter 21(48): 485001 (2009)

[19] Persson B N J. Contact mechanics for randomly rough surfaces. Surf Sci Rep 61(4): 201-227 (2006)

[20] Ronsin O, Coeyrehourcq K L. State, rate and temperaturedependent sliding friction of elastomers. Proc Roy Soc A: Math Phys Eng Sci 457(2010): 1277-1294 (2001)

[21] Chateauminois A, Fretigny C. Local friction at a sliding interface between an elastomer and a rigid spherical probe. Eur Phys J ESoft Matter 27(2): 221-227 (2008)

received his Ph.D. degree in tribology from University of Twente. His current position is a lecturer and researcher in Islamic University of Indonesia. His research interests include tribology of elastomeric material and biotribology. 